\title{
Un enfoque hermenéutico fenomenológico sobre la perspectiva narrativa \\ Daniel Chamberlain
}

\author{
Queen's University
}

n los años sesenta, Scholes y Kellogg (1966) definieron la perspectiva en
la narrativa como la uesencia del arte narrativo» (240).' La perspectiva juega un papel esencial en la producción y en la recepción de textos literarios y se ha convertido en un tema importante para las teorías que surgen del proceso narrativo. Diferentes enfoques teóricos han buscado definirla en términos de persona, punto de vista, reflector, voz, conciencia central y focalización. A pesar de la gran variedad de enfoques y del debate continuo en cuanto a la naturaleza de la perspectiva en la narrativa, la mayoría de las teorías concuerdan en que la perspectiva está de una manera u otra involucrada en el proceso más general de la percepción. Sin embargo, con gran frecuencia se entiende la percepción exclusivamente en términos del impacto en los sentidos de los estímulos que proceden del mundo exterior. Entendida así, como un impacto sobre nuestros sentidos, la percepción se encuentra muy a menudo reducida a una sola de sus dimensiones esenciales. Este concepto de información que procede del mundo exterior y que cae en una mente en blanco, da a la dimensión espacial de la percepción una autoridad supuestamente objetiva e innegable a la cual muchas veces los teóricos acuden. El resultado es que ignoran la dimensión temporal de la percepción que involucra la selección de información significativa en la experiencia del mundo. Así, estos teóricos se acercan a la noción de la perspectiva en la narrativa partiendo de una definición del lector como alguien pasivo y presuponen un autor o un texto activo. Cuando se entiende la perspectiva como parte íntegra del proceso global que es la percep-

\footnotetext{
- Poligrafías 1 (1996) 83-103

Poligrafias. Revista de Literatura Comparada, División de Estudios de Posgrado, Facultad de Filosofía y Letras, Universidad Nacional Autónoma de México, Ciudad Universitaria, México 04510 D. F.

Tel. (525) 622 1835(6). Fax (525) 622 1801; 616 0047; 6221826.
} 
ción, no se puede presuponer una definición pasiva del lector. La percepción es una apertura por medio de la cual se correlaciona nuestra conciencia del mundo con una conciencia de nosotros mismos. Es, según Maurice MerleauPonty, una «recreación o reconstrucción del mundo» que se lleva a cabo a cada momento $(1962,207)$. Entendida así, se pueden correlacionar la dimensión espacial, la dimensión temporal de la percepción al lenguaje y la experiencia. Entendiendo la percepción como una apertura hacia una dialéctica de espacio y tiempo en el proceso de la conciencia, se pueden aclarar algunas cuestiones que subyacen la noción de perspectiva en general para poder entender el papel que desempeña en la lectura de textos narrativos.

Este entendimiento de la perspectiva en la narrativa empieza con el concepto de Hans Georg Gadamer de wirkungsgeschichtiches Bewusstsein o conciencia bajo los efectos de la historia (1975, 305). Para Gadamer, la conciencia está unida con el mundo histórico a través de una jerarquía de cuatro niveles relacionados: lenguaje, percepción, experiencia y concepto (314). Considerando que la narrativa presupone el lenguaje y que la perspectiva es una parte íntegra de la percepción, la perspectiva en la narrativa procede del lenguaje y de la percepción y lleva a una experiencia literaria; en última instancia, es esta experiencia literaria la que precede a todas nuestras teorias.

La filosofía de Gadamer parte de la noción ontológica en la que el ser es lenguaje (443). Siguiendo a Humboldt, él afirma que hay una relación íntegra entre el lenguaje y el mundo y que el lenguaje, como energeia, es lo que da al ser humano el poder de tener un mundo y de poder comunicarlo (Humboldt 49 y Gadamer 401). Tanto la realización del lenguaje por medio de un diálogo como el entendimiento del mundo desde una tradición, constituyen la preocupación primordial de Gadamer. Para Gadamer, ni el diálogo ni el entendimiento pueden ser reducidus a diferencias puramente formales. Una conversación entre un «tú» y un «yo» busca un acuerdo en cuanto a algún objeto o asunto.

Gadamer estructura el concepto de conversación en términos de una dialéctica de pregunta y respuesta. Una pregunta nace al darnos cuenta de que hay algo que no sabemos. De este no-saber fundamental y del «saber» que no sabemos, surge una pregunta que se extiende en múltiples direcciones buscando varias posibles respuestas. Este estado inicial, marcado por una falta de dirección determinada, se resuelve cuando la pregunta se concentra en una dirección específica que señala una respuesta específica. La negatividad en que nace la pregunta no es un vacío absoluto. Este vacío está impregnado de tradición, y la hermenéutica de Gadamer enfatiza la importancia que tiene el escuchar las voces de la tradición que llevamos en el lenguaje. Las voces de la tradición dirigen las actitudes de los interlocutores en el diálogo y guían el sentido de sus preguntas al buscar respuestas. Cada respuesta sensata a la vez refleja la pregunta de donde procede. Hay entonces una dirección centrífuga primaria que caracteriza la 
pregunta y una dirección centrípeta secundaria que se refleja desde la respuesta. Estas dos direcciones en conjunto guardan el sentido de la pregunta.

Para Gadamer entonces el modo de ser que se puede entender es el lenguaje como energeia. Para Merleau-Ponty, ser es percibir entendiendo la percepción como un extenderse hacia el mundo y como un asir el mundo por medio de nuestro cuerpo en acción. El lenguaje y la percepción comparten estructuras y características semejantes. Asi como una pregunta se dirige por medio de un sentido hacia una respuesta al entrar en diálogo con otros, así el cuerpo se abre por medio de los sentidos hacia un objeto y entra en un diálogo con el mundo. Para Gadamer el centro de la pregunta es un no-saber y para Merleau-Ponty el corazón de la percepción humana es una región anterior a nuestros cinco sentidos, un nivel de no-sentido.

Maurice Merleau-Ponty caracteriza este nivel de no-sentido primordial en términos de una fe primaria, una creencia en el mundo marcada por el lenguaje y por una tradición. Este nivel de fe y de creencia se encuentra a un nivel más profundo que las dualidades y dicotomías que la mente racional tiene que emplear para definirlo. Este no-sentido ek-stática se extiende hacia el mundo a través del tiempo y su punto de partida es un ahora eterno (Merleau-Ponty 1962,422 ). Nuestros sentidos median este nivel primario del no-sentido y el mundo que nos rodea. Inicialmente, no hay una división de los sentidos en cinco unidades discretas. No se dividen en términos del tacto, sabor o gusto, olfato, oído, y vista sino yacen en conjunto dentro de un campo de comunicación intersensorial. Entre el no-saber y el mundo se encuentra un campo de sentidos indeterminados en comunicación sinestética. Solamente cuando iniciamos una acción, la percepción se concentra en un sentido específico y se abre hacia un objeto en el mundo por medio de ese sentido. Es en este momento de sinergia que se hace posible una dialéctica de «sujeto» en el tiempo y de «objeto» en el espacio dentro de una situación común. Un punto de partida, o «aquí,» se realiza en conjunto con un horizonte «allá» y se acomoda adentro del horizonte un objeto en primer plano y otros en un trasfondo. La perspectiva es todo lo que media el polo subjetivo y el polo objetivo de esta dialéctica. La perspectiva es inseparable del sentido en que la percepción se concentra en el acto subjetivo de extenderse hacia el objeto en el mundo. Diferentes sentidos enfatizan diferentes cualidades. El sentido auditivo está íntimamente relacionado con el punto de partida del sujeto en el lenguaje y en una tradición mientras que el sentido de la vista goza de mayor libertad para explorar el espacio y analizarlo con gran precisión. La distancia es más el resultado del poder que el cuerpo tiene de captar un objeto que una medida objetiva calculada por medio de una regla arbitraria.

El espacio existe como una relación entre cosas que surge de la situación creada por el cuerpo en acción. La noción de altura y de ancho se logra por 
medio de la profundidad y la profundidad es la dimensión primaria y más subjetiva del espacio. Así como la fe primaria permite que un objeto exista como una unidad, así la profundidad permite que un objeto se conforme a una unidad consistente. La profundidad es la mediación del espacio y es desde la profundidad de nuestro ser que proyectamos nuestras acciones hacia un mundo de relaciones significativas. Una vez que logramos la existencia de un objeto en el espacio, ese objeto refleja la profundidad del proceso de su percepción.

El tiempo es la subjetividad que el espacio, entendido como relaciones significativas, presupone. Es la cohesión del espacio. Como la noción agustiniana de intentio y distentio animi, el tiempo es ambos un ahora omnipresente y una sucesión de eventos pasados, presentes y futuros (San Agustín en Ricoeur 1984, 1:29). Como la energeia, que es el lenguaje, fluye de sí mismo y está marcado por una dialéctica de cohesión y distanciamiento, de un sentido y no-sentido. El tiempo es la profundidad de una tradición compartida que reúne elementos diversos dentro del alcance de un solo punto de vista.

La perspectiva es la mediación entre toda interacción de sujeto y objeto en el tiempo y espacio y esta mediación se lleva a cabo en dos direcciones. Por un lado ofrece el medio por el cual se alcanza un objeto y por el otro, ofrece una reflexión sobre el proceso mismo. Así como una respuesta refleja su pregunta, una perspectiva nos lleva al punto de partida de donde se lleva a cabo la observación y puede llevar al que observa a un entendimiento de sí mismo. Las nociones de perspectiva y de sentido son inseparables. La palabra «sentido» tiene un valor polisemántico amplio. Como la palabra sens en francés, la palabra «sentido» significa «significado,» «dirección,» «actitud,» además de «tacto,» «visión,» «oído,» «olfato,» «gusto.» Merleau-Ponty califica la perspectiva que se inclina hacia una exploración reflexiva, analítica y precisa, como una "perspectiva artificial» $(1964,48-50)$. El sentido de la vista se presta a esta perspectiva artificial que explora las relaciones lineales del espacio. Una perspectiva que se inclina hacia el reporte de una experiencia común o una tradición compartida a través del diálogo, Merleau-Ponty la llama una perspectiva «natural» o «común» (48-50). El sentido auditivo se presta a esta perspectiva común y a su reporte de circunstancias en el tiempo. Un cambio de sentido es un cambio de dirección que implica un cambio de actitud, un cambio de «perspectiva» y ese cambio está correlacionado a una modificación en la experiencia de estar en el mundo.

El lenguaje y la percepción nos llevan a experiencias por medio de eventos significativos. En lugar de evitar los aspectos sin sentido de fenómenos semánticos y en lugar de evadir el conflicto de interpretaciones que resulta, Paul Ricoeur ha celebrado la naturaleza polisemántica del lenguaje como discurso, la tensión dinámica que es la metáfora, y la experiencia hermenéutica productiva. Para Ricoeur, la metáfora es la tensión que da lugar a una experiencia que 
vibra con nuevos sentidos en un evento de descubrimiento que surge precisamente de una impertinencia semántica o no-sentido (1977, 247-248). Es una relación dinámica que afirma que algo sí es así y que simultáneamente no es así. El discurso literario se identifica como esa referencia bifurcada. El sentido de las palabras se señala en dos direcciones, una hacia el mundo histórico o primordial que nos rodea y la otra hacia el mundo metafórico o secundario del texto.

Este enfoque dialéctico que entiende la narrativa como una experiencia, reúne el carácter de tiempo y tradición, lo que Ricoeur llama «muthos,» con la estructura, o «mimésis,» de la creación, configuración y recepción de la narrativa (244 y 245). Si el distentio y el intentio animi, o muthos, es la unidad temporal narrativa, mimésis es una imitación más objetiva de una acción que refleja esa unidad. Muthos es la cohesión temporal de la narrativa, una relación entre sus eventos que se encuentra reflejada en los tres momentos interrelacionados de mimesis. El primer momento de mimesis o prefiguración es un acto por parte de un autor competente que lleva a la creación de un texto. La configuración resultante, o mimesis dos, refleja las condiciones de su creación pero no está limitada a las intenciones subjetivas del autor. Mimesis tres o refiguración es la participación activa de lectores en la realización del texto. Un autor se apropia de su mundo para crear un texto y el lector realiza el texto por medio de un proceso de apropiación. ${ }^{2}$

La apropiación, como la perspectiva, pone en juego sentidos centrífugos y centripetos. Para Ricoeur apropiación es la relación dinámica de integrar algo que era inicialmente ajeno y extraño. A esta acción asimilativa, sin embargo, le acompaña un distanciamiento esencial que siempre mantiene aspectos de lo ajeno fuera de nuestro alcance. No nos es posible percibir un texto entero a la vez sino que siempre quedan aspectos por descubrir. Siempre quedan aspectos fuera de nuestro alcance porque percibimos el texto desde un punto de partida específico en el mundo y en el tiempo. El evento que es la apropiación de un texto descubre no solamente otro modo de estar en el mundo sino revela por medio de su perspectiva el punto de partida del lector. Este momento de descubrimiento del otro y la revelación de si mismo es la experiencia fundamental que la narrativa nos ofrece (Ricoeur 1981, 182-193).

La noción de perspectiva entonces, juega un papel clave en la dialéctica de pregunta y respuesta al nivel de lenguaje, un papel intrínseco en la dialéctica de cuerpo y mundo al nivel de la percepción, y un papel esencial en la interacción entre lector y texto en la experiencia de la narrativa. Cada una de estas relaciones está marcada por una dirección que procede del primer término al segundo y, luego, por una dirección que procede desde el segundo al primero como si reflejara el proceso mismo. No se puede divorciar el concepto de perspectiva en la narrativa de estas nociones generales de lenguaje, percepción 
y experiencia. Al grado que la narrativa es lenguaje, es conciencia bajo los efectos de la historia. Al grado que perspectiva es sentido, es percepción. Al grado que la perspectiva en la narrativa es esencial a la narrativa, es vital en la interacción entre lector y texto en una experiencia humana del tiempo y del espacio. Un texto es una configuración del discurso hacia la cual los lectores se extienden al ponerla en juego (Ricoeur 1984, 1:53). La experiencia hermenéutica, o sea el escuchar, el cuestionar y el encontrar respuestas en un texto, hace de la perspectiva en la narrativa, una relación, una intersección, que surge entre interlocutores en el diálogo que es su realización. $\mathrm{Si}$, como lo afirma Wolfgang Iser, la perspectiva es una visión canalizada que establece el modo específico de acceso al objeto intencionado (Iser 1978a, 113), hay que aclarar que hay dos canales en la interacción: uno que lleva del texto hacia la conciencia histórica del lector y el otro que se extiende del lector hacia el texto. Esta cualidad estereoscópica de la perspectiva en la narrativa exige que cualquier estudio de su función tome en cuenta las dos direcciones de los canales.

Si examinamos las teorías más importantes que han tratado la perspectiva en la narrativa podemos entenderlas en términos de diferentes momentos en un proceso conceptual que oscila entre un polo temporal y subjetivo de relaciones personales, y una idea más objetiva de un reflector central o punto de vista en el espacio. Aquel polo desarrolla la noción de perspectiva en términos de una relación entre personas en un diálogo, mientras este polo busca eliminar la noción de persona en favor del espacio estructurado de un mundo narrativo. La diferencia en el sentido o dirección de la oscilación ha formado la base de las diferencias entre las teorías que buscan definir la perspectiva en la narrativa. Henry James, en su rechazo del privilegio romántico de la primera persona y en su insistencia en definirla como el empleo de un solo centro, así como Percy Lubbock, quien ve el punto de vista en términos del método que establece la apariencia de un cuento, reconocen pero menosprecian aspectos subjetivos en favor de consideraciones objetivas de forma (James 1934, 317 y 320, y Lubbock 1957,251 y 265). En su respuesta a Lubbock, E. M. Forster resalta la capacidad que tiene el autor de manipular al lector y destaca la relación personal que establece el autor con sus personajes (78). Norman Friedman también considera el punto de vista como una relación estética entre el autor y su obra (109). M. H. Abrams (1957) regresa a un criterio más objetivo cuando busca establecer dos categorías formales, la de narraciones en primera persona y las de narraciones en tercera persona (134). Wayne C. Booth reacciona en contra de Abrams al afirmar que las categorías de primera y tercera persona no nos dicen nada de importancia al menos de que establezcamos cómo es que ciertas cualidades específicas del texto llevan a efectos específicos en el lector (Booth 1961,150 ). Con Booth el péndulo gira de nuevo hacia el polo subjetivo, pero ahora es una subjetividad ubicada en el lector no en el autor. 
El advenimiento del formalismo y del estructuralismo trajo consigo una perspectiva objetiva de nuevo. Jurij Lotman define el punto de vista como la jerarquía de relaciones y sus orientaciones en un sistema que crean un mundo en un sujeto o en una conciencia capaz de engendrar estructuras semejantes $(1977,265$ y 268). Fiel a las oposiciones binarias formalistas, Lotman lleva al lector a una interpretación correcta y verdadera que coincide con el punto de vista del texto o a una interpretación incorrecta que se opone a ello. Recientemente la narratología de Gerard Genette ha buscado explicar la perspectiva en términos de focalización o un regulador de información. Genette entiende la focalización en términos de una metáfora «espacial» $(1980,162)$. La focalización depende de la "distancia» que separa al lector de la «información narrativa por su precisión» (162). Shlomith Rimmon-Kenan explica la focalización en términos de factores textuales también y descarta la noción de un lector histórico como un concepto «extremo» $(1983,119)$. La focalización de Mike Bal se acerca más a la noción hermenéutica de perspectiva pero para ella es la manera en que un texto manipula al lector. En otras palabras, invierte la dirección de prioridad entre el lector y texto. El texto es activo y manipula a un lector pasivo. Si Bal afirma que hay textos en que el espacio mismo es el objeto de presentación, Ricoeur afirma que hay cuentos en que es una experiencia del tiempo mismo la que se quiere llevar a cabo (Bal 1985, 95 y Ricoeur 1984, 2:101).

Podemos decir, entonces, que una vez que las intenciones subjetivas del autor ya no sirvieron como criterio exclusivo para establecer la perspectiva de un texto, la teoría literaria buscó en las estructuras del mundo del texto la manera de mostrar cómo esas estructuras reflejan una focalización narrativa. Sin embargo, últimamente la teoría literaria ha dejado de basarse en relaciones espaciales aparentemente objetivas y se ha movido una vez más hacia la noción subjetiva de persona. Esta re-introducción de la dimensión personal ha cuestionado la validez de conceptos objetivos de focalización narrativa. Como respuesta a este interrogatorio, por lo menos tres posturas hermenéuticas importantes han introducido de nuevo al lector histórico. Robert Weimann reintroduce la perspectiva del lector al nivel de la prefiguración del texto cuando insiste que el lector tome en cuenta las condiciones socio-económicas de la producción del texto $(1975,20)$. Wolfgang Iser introduce al lector al nivel de la configuración del texto al aclarar cómo diferentes lectores reaccionan a estrategias textuales para posteriormente reaccionar en contra de normas tradicionales (1978b, 103 y 106). Finalmente, Hans Robert Jauss reintroduce al lector al nivel de la refiguración. A diferencia de Weimann, Jauss estudia cómo la perspectiva estética de un momento en la historia encuentra la perspectiva estética de una época previa (169). Aunque los tres consideran la perspectiva del lector, Weimann da prioridad a intereses socio-cconómicos en el mundo 
del autor, Iser da prioridad a la noción de ficción como una reacción estructurada en contra de normas sociales y Jauss tiende a divorciar una percepción estética o interpretativa por un lado y el proceso de la percepción cotidiana por el otro. Aunque los tres comparten una cuestión común, cada uno tiende a enfatizar diferencias en lugar de semejanzas al ofrecer respuestas.

Hace falta, entonces, acercarse a la perspectiva en la narrativa por medio de una coordinación más balanceada entre diferencias $y$ semejanzas de tiempo $y$ espacio, entre lector y texto, entre el mundo del lector y el del autor. Dada la complejidad del lenguaje, la percepción y la experiencia, el reto es de buscar categorías sencillas y aplicables. La frustración que expresan teóricos como Rimmon-Kenan cuando tratan de entender lo que se entiende por «lector» es justificada. Tenemos al «lector actual» (Van Dijk y Jauss), el «superlector» (Riffaterre), el «lector informado» (Fish), el «lector ideal» (Culler), el «lector modelo» (Eco), el «lector implícito» (Booth, Iser, Chatman, Perry) y el «lector codificado» (Brooke-Rose), para mencionar unos cuantos (Rimmon-Kenan 118). A pesar de las diferencias entre estos conceptos de lector, todos presuponen una primera persona situada en el mundo informada por el lenguaje, un yo que percibe un mundo y que tiene una experiencia con el mundo del texto por medio de un diálogo de pregunta y respuesta con una voz narrativa. Cada lector es, en última instanciia, un yo que tiene una experiencia del texto y que entra en una relación con una voz narrativa o tú y un mundo narrativo, o sea, un él, ella y ello. Aunque un punto de partida para el lector en esta relación interpersonal puede estar implícito en el texto, la perspectiva personal que percibe el texto no es el resultado exclusivo de las estrategias del texto.

La perspectiva en la narrativa es una relación que se lleva a cabo entre las técnicas y las estrategias del relatar por un lado y las perspectivas tradicionales e individuales del autor implícito y el lector en la creación y la recreación del texto por el otro. La perspectiva en la narrativa es subjetiva al grado de que procede del carácter temporal de la experiencia humana y es objetiva al grado de que estructura la actividad de narrar un cuento para crear un mundo narrativo. Se logra la perspectiva en la narrativa al poner en juego una variedad de facetas interrelacionadas que se ubican dentro de tres dimensiones fundamentales en el proceso narrativo. Estas dimensiones son la dimensión subjetiva del tiempo; la dimensión objetiva de relaciones espaciales y la dimensión fenomenológica de percepción. No se puede limitar la perspectiva al polo temporal ni al polo espacial porque es un proceso de mediación. Las facetas de la perspectiva entran en juego para mediar estas dimensiones en cada momento del proceso narrativo: en la prefiguracion del autor, en la configuración resultante y en la refiguración por parte del lector.

Si nos acercamos al texto desde su lado noemático podemos dividirlo en consideraciones de voz por un lado y mundo narrativo por el otro. La voz 
narrativa reúne seis facetas: persona, dramatización, alcance, confiabilidad, secuencia temporal y duración temporal. Entiendo la primera faceta, persona, en términos etimológicos como lo explica Benveniste. «Persona» aquí quiere decir «figuración» (personce) y no máscara en el sentido en que se usa en el teatro (Benveniste 1966, 1:225). La primera y segunda persona constituyen un polo más subjetivo en el discurso mientras que la tercera persona (o más bien no-persona porque no figura en la situación del diálogo) ofrece un polo objetivo (1:231). Un punto de partida colocado en la tercera persona o más bien en la no-persona facilitaría una observación objetiva de las facetas mismas. La voz pasiva, por ejemplo, busca eliminar la persona al invertir el orden de sujeto-objeto. Busca una autoridad por medio de una objetividad aparente que, sin embargo, presupone personas en un diálogo. El pronombre con que la voz narrativa lleva a cabo su relato establece necesariamente una relación en dos direcciones: una hacia el mundo narrativo y la otra hacia el lector. El uso de la primera persona tiende a crear un tú en el lector; el uso del tú crea el punto de partida de un yo. El uso de un él, ello o ella crea un punto de partida compartido por tú y yo, o sea, nosotros. Al escoger el pronombre, uno escoge una figuración que afectará la perspectiva narrativa a todo nivel. El uso de un pronombre muy impersonal como uno o nadie creará un punto de partida abstracto, artificial, y reflexivo. Encontramos un buen ejemplo de este uso artificial en las líneas con que Jorge Luis Borges empieza «Las ruinas circulares:»

Nadie lo vio desembarcar en la unánime noche, nadie vio la canoa de bambú sumiéndose en el fango sagrado, pero a los pocos días nadie ignoraba que el hombre taciturno venía del Sur. ... (1980, 1:435)

Podemos entender la segunda faceta, «dramatización,» como el grado en que la voz entra en las relaciones de causa y efecto de la acción del cuento. Así puede estar situada en un extremo como participante en primera persona o en el otro extremo como una fuente escondida de revelación o, claro, en cualquier punto entre estos dos extremos. El grado de enredo o de participación en la acción que tiene un personaje ayuda a establecer la estructura de valores en el texto.

El alcance es el poder de síntesis que tiene la voz, o sea, qué partes potenciales de la dimensión temporal y la espacial puede reunir en una unidad consistente. Incluye el punto de partida o under-standing desde donde se seleccionan aspectos para figurar algo y también lo que coloca en primer plano y lo que establece como trasfondo. El alcance de la voz crea el espacio interior y exterior y representa la capacidad de presentar experiencias del pasado $y$ posibilidades futuras desde su presentación presente. El lector, claro, trata de reunir aspectos de su percepción y experiencia con la narración. Cada lector llena los huecos que deja la voz con las percepciones y experiencias que tiene a su alcance desde la tradición en que vive y del mundo narrativo que ha 
formulado. El hueco más grande en todo texto es el hueco entre el final y el principio de un texto. El final proporciona para el lector el punto de partida con mayor alcance del texto.

La confianza que tenemos en una voz no constituye una categoría distinta sino una dirección de movimiento. La voz o el lector puede construir una narración en una secuencia de causa-efecto o invertir el orden en términos de efecto-causa y de-construir o socavarla por medio de la paradoja e ironía. Esta inversión del orden ilumina las facetas en sí pero deja la figuración que presuponen en la oscuridad. Tanto la hipérbole como la atenuación son estrategias que reflejan una voz que invierte el sentido de la perspectiva.

Con su obra Temps et Récit, Ricoeur ha dejado en claro la relación estrecha entre el tiempo y la narrativa. Para la perspectiva en la narrativa es esencial. El tiempo que la voz toma para relatar un evento y la dirección que toma en crear una secuencia de eventos son fundamentales para estructurar una experiencia. Sin embargo, durante una lectura el lector normalmente no tiene todo lo narrado presente. El carácter de la experiencia que cada lector tiene de la narración depende de su memoria de la secuencia de eventos y de la duración de las preguntas que dirige al texto. Toda la perspectiva de un texto es temporal tanto en el sistema cronológico que ofrece la voz, como en las preguntas que nacen en el lector, como en el tiempo que toma el lector en encontrar o crear respuestas. El movimiento del lector a través del texto no es únicamente un movimiento errante que va vagando por el texto como lo afirma Iser (1978a, 112). El movimiento deambula según la individualidad del lector pero es dirigido por las estrategias del texto por un lado y por la tradición en que vive por el otro. Cada comunidad de lectores y cada tradición de crítica literaria tiene el derecho de retar y cuestionar la validez de la dirección que una perspectiva crítica toma. Cada crítico tiene que buscar el acuerdo de una comunidad de críticos en cuanto a la validez de su interpretación del carácter y de la estructura que dirige su lectura.

Para entender la figuración de una narración de tiempo, el dibujo de una elipse (Figura A) y la noción de elipsis sirven de ayuda. La narración de tiempo resulta de una perspectiva compartida o común que une al lector con la voz. La figura de una elipse traza un círculo $P$ y la suma de la distancia entre los dos Figura A focos fijos $F$ y $F^{\prime}$ es una constante. En 
una narración de tiempo la experiencia de todos los eventos o puntos $P$ resulta de la suma de las distancias entre la voz o $F-P$ y los eventos y el lector y los eventos $F^{\prime}$-P. Esta relación cumulativa de asimilación sitúa al lector y la voz en el ahora de la situación en que se lleva a cabo el relato alrededor de ellos. A través de la historia y a través del mundo hoy en día, la distancia entre la voz que narra como un punto fijo y el lector como otro punto fijo, varía pero los dos siguen dentro del marco de una lengua y comparten el momento presente de la situación en que se lleva a cabo la narración. Etimológicamente, elipse y elipsis provienen de la misma raíz griega. Elipsis es una omisión significativa y es significativa al grado en que la secuencia de eventos pasados se relaciona al entendimiento de un lector en el presente y al grado en que constituye un advenimiento de posibilidades futuras. Esto no depende únicamente de la perspectiva del lector. Depende de la relación que guarda con la perspectiva que ofrece la voz y de lo que el lector interpreta o tigura en términos de significado. Una elipsis es un vacío en el texto que está lleno de significados personales e intersubjetivos. Una elipsis es una nada que es algo, una ausencia que presenta un significado. La elipsis reúne la tensión fundamental que define la metáfora como tropo literario primordial. La elipsis pide la presencia del lector y el lector, como lo ha sugerido Iser, llena el vacío (1978a, 97 y 112). El cuarto capítulo del Lazarillo de Tormes, por ejemplo, no consta de más de cuatro oraciones pero representa una elipsis de ocho días significativos. Harry Sieber ha llevado a cabo un estudio profundo que llena esta elipsis con catorce páginas de interpretación (1978, 45-59).

Como parte de un proceso de percepción, la perspectiva en la narrativa reúne el tiempo y el espacio en el mundo imaginario del texto. Al hablar de secuencia y de duración uno ya habla de relaciones espaciales. El mundo narrativo emerge de la intersección de la perspectiva del lector y de la voz narrativa. Se trata de un mundo de relaciones entre fenómenos. La relación entre objetos de importancia simbólica tanto para el lector como para la voz modificará la estructura de la perspectiva narrativa. ¿Qué mundo resultará de la perspectiva de $L a$ guerra de los mundos de H. G. Wells? Depende del lector. Si el lector es un inglés del siglo veinte será un mundo fantástico de pura ciencia ficción. Pero si es un mexicano con plena conciencia de lo que hæ de haber sido la conquista, si es un mexicano quien ha heredado lo que León-Portilla y Garibay han llamado la «visión de los vencidos,» ¿qué tan fantástico, qué tan ficticio sería ese mismo mundo narrativo?

El mundo narrativo es el espacio que media y que separa los objetos presentados en el primer plano y los que forman el trasfondo. Son también las relaciones que unen y separan a los personajes en una jerarquía social. En esta dimensión del mundo narrativo la perspectiva del lector se abrirá a estructuras que reflejan el carácter de las seis facetas de la voz. Aparte de objetos en primer 
plano, hay que considerar los diferentes tipos de personajes, los papeles que representan, cómo los privilegia la voz, y por último el grado de confianza que le otorgamos a un personaje.

El tipo de personaje depende de la voz pero también de la relación que guarda con el lector. Si es héroe o villano depende de dónde se coloca un personaje en un momento específico en el mundo del texto y depende de la perspectiva del lector ya que juicios éticos provienen del mundo histórico. También el tipo de personaje resulta de las estructuras sociales del mundo narrado y de la percepción del lector, quien entiende ese mundo por medio de una perspectiva figurada por una tradición social. Si el personaje es un arquetipo común o si constituye un prototipo individual dependerá de la interacción de distancia y mediación entre ese personaje y su mundo, por un lado, y el entendimiento que tiene el lector de ese mundo por el otro.

La relación que se guarda entre la voz que narra, el lector que cuestiona y el tipo de personaje es inseparable del papel que el personaje juega en las relaciones de causa y efecto que constituyen la acción del relato y sus eventos. Los personajes son figuraciones de experiencias en el mundo narrativo. Es por medio de estas experiencias que la voz, como lo explica Jean Pouillon, «trata de dar al lector el mismo entendimiento de los personajes que ese lector tiene de sí mismo» (citado en Ricoeur 1984, 2: 183-184). Este grado de participación en las relaciones de causa y efecto de la acción tendrá un impacto en la experiencia que el lector tiene del mundo narrativo. A la vez las experiencias que tiene el lector del mundo en que vive afectarán no solamente la manera en que llena las elipsis que encuentra sino de alguna manera el grado de intensidad del interrogatorio que aplica a cada papel.

El privilegio del que goza un personaje depende del grado en que la voz reúne múltiples aspectos del espacio y tiempo para crear una figura con estatura, consecuencia y profundidad. El personaje no es privilegiado si la voz no puede o no quiere introducir la tercera dimensión de profundidad. Un personaje privilegiado es uno que ha sido desarrollado plenamente y que está colocado en primer plano. No se puede aislar esta relación entre partes y la unidad entera por medio de una consideración exclusiva de eștrategias textuales. Un personaje supuestamente pleno puede resultar de poca profundidad para un lector cuyas experiencias le han dado una profundidad mayor.

La confiabilidad de los personajes depende de su enajenación de los eventos en el mundo narrativo y del grado de paradoja que introduce en las estructuras de causa y efecto. Un personaje no goza de nuestra confianza si se encuentra distante de los eventos y si aparentemente quiere socavar la dirección en que se mueve el relato. Con frecuencia, un personaje estereotipo participa en las relaciones de causa y efecto de una manera predecible y depende plenamente del sentido o dirección que tiene la acción del texto. Un personaje enajenado 
tiene un mayor grado de independencia del sentido o dirección en que se lleva a cabo la acción. Goza de mayor libertad para hablar independientemente y con frecuencia tiene el privilegio de introducir mayor abstracción porque sabe más. La paradoja que introduce un personaje no confiable mueve al lector desde un dominio del sentido del texto hacia la incertidumbre y hacia una falta de sentido definitivo. La paradoja lo lleva hacia un no-sentido y es este no-sentido que invita mayor participación por parte del lector.

Las relaciones entre objetos en primer plano y los del trasfondo resultan en la escena de la acción. Como en el caso de secuencia y duración temporal, lo que está en primer plano y lo que constituye el trasfondo depende del modo de percibir el mundo y del punto de partida de la voz. Son estas facetas las que han ofrecido un punto de partida para muchos de los enfoques teóricos que entienden «perspectiva» primordialmente en términos de punto de «vista.» Todas las relaciones de objetos en primer plano y de objetos en el trasfondo presuponen un aquí de la voz $y$ un aquí del lector. La distancia que media entre estos dos puntos varía. A diferencia de un ahora omnipresente que se comparte en la experiencia común del tiempo, el aqui de un punto de partida ya involucra dos puntos necesariamente separados que se pueden compartir solamente por medio de un

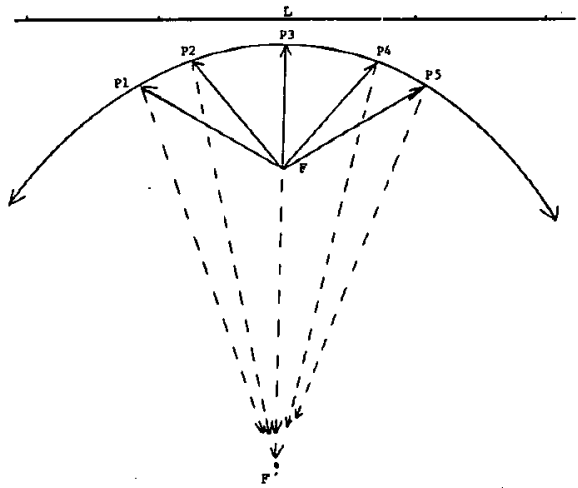

Figura B análisis abstracto por reflexión intelectual.

Si la figura de una elipse puede ayudar en entender un texto que trata del tiempo, entonces la parábola de la Figura $\mathrm{B}$ puede ayudar en entender un texto que trata del espacio. Una parábola es la curva delineada por todos los puntos $P$ cuyas distancias de una línea fija $L$ equivalen a la distancia de un punto fijo $F$ no en la línea $L$. De nuevo los puntos $P$ representan los eventos narrados y la linea $L$ representa lettres o langue. La voz se encuentra de nuevo en el punto $F$ al igual que en la elipse. Aquí no se habla del lector en el punto $F^{\prime}$. La parábola se forma cortando la elipse a la mitad y enajenando al lector, $F^{\prime}$, quien ya no figura en el relato. Ahora se coloca a espaldas de la voz quien le atribuye un papel pasivo. La voz se dirige lejos del lector hacia el mundo de personajes quienes reflejan su presencia indirecta. Este mundo emerge entre el punto de partida de la voz y las estructuras de la lengua escrita que la voz explora para estructurar un espacio. En términos de Northrop Frye esta estructura es seme- 
jante a la que resulta de la voz del poeta lírico quien «le da la espalda a sus oyentes, aunque él puede hablar en lugar de ellos, y aunque ellos pueden repetir algunas de sus palabras después de él» $(1957,250)$. En esta estructura la voz refleja o más bien rebota de las paredes cóncavas del mundo del texto por medio de las voces de los personajes creando un eco polifónicọ. En lugar de una secuencia circular de eventos el lector puede encontrar un movimiento pendular oscilando de un extremo a otro en una dicotomía que llega a descansar en el centro. Este movimiento alternante y pendular revela la tensión entre el tiempo abstracto del reloj por un lado y el tiempo de la experiencia por el otro. La parábola personifica el espacio y parece que el espacio tiene una voz propia. La objetividad aparente de las voces parece proceder del texto mismo y, como toda estructura parabólica, invierte el orden de las cosas dejando al lector con poco en qué confiar. Si la relación temporal de la elipse invita al lector a participar en la acción por medio de su refiguración, las relaciones espaciales de la parábola reflejan y rebotan sobre la perspectiva del lector, sobre el proceso de percepción mismo, y apuntan hacia su voluntad de actuar y de jugar una parte activa en la exhibición que es la narración.

Habiendo ya considerado las facetas de la voz narrativa y las del mundo narrativo queda por considerar el punto de partida vital del lector en el mundo constituido por el lenguaje. Si la perspectiva en la narrativa ofrece el canal o el medio por el cual el lector se extiende hacia el texto por un lado, y por medio del cual el proceso de percepción y un autoentendimiento se revelan por el otro, entonces es a través de las mismas figuras del lenguaje que se puede desarrollar una tipología de diferentes modos de entendimiento. De acuerdo con la naturaleza figurativa de ambas, persona y metáfora, hace falta más bien una tropología de perspectivas del lector en que cada tropo sugiere la manera en que lectores figuran su mundo y el texto. Hace falta una tropología de las maneras en que le damos sentido al texto, de las perspectivas por las cuales se percibe el texto y por las cuales se revela cada lector. La tipología del discurso desarrollado por Hayden White es significativa aquí. White propone una tipología , o más bien tropología, del discurso que media entre las figuraciones poéticas de la realidad y la comprensión noética que tiene un lector de esa realidad. Siguiendo a Kenneth Burke, White propone una tipología de cuatro niveles empezando con la metáfora, pasando a la metonimia, luego a la sinécdoque y finalmente a la ironia $(1978,5)$. El primer nivel se caracteriza por una tensión en la cópula y el último nivel lleva a una reflexión sobre el proceso que establece el orden de las cosas. No se trata de niveles discretos sino de un proceso que se extiende como una hélice y que coordina perspectivas diferentes y semejantes por medio de distintos grados de semejanza y diferencia simultáneamente. El caracterizar las experiencias en términos metafóricos es caracterizarla en términos que afirman una semejanza en una diferencia e, implícitamente, 
una diferencia en una semejanza. Se asocia con el desarrollo del niño en el campo de investigación ontogenética y con las sociedades supuestamente más primitivas. ${ }^{3}$ En esta conciencia de identidad, dicotomías y conceptos discretos tales como: significado frente a significante, la vida versus la muerte, lo bueno frente a lo malo y lo correcto frente a lo incorrecto, tienden a confundirse el uno con el otro. En la configuración metafórica, el individuo no reta la autoridad de la tradición. En el tiempo predomina un ahora perpetuo y se coloca el aqui inmediato en primer plano.

Una noética metonímica, por el otro lado, es reductiva y mecánica. Reduce el mundo a relaciones extrínsecas de una parte frente a otra y comprende las experiencias en términos de leyes que atan un fenómeno a otro en relaciones de causa y efecto. De esta conciencia metonímica emergen las categorías conceptuales necesarias para la reflexión abstracta, teológica y académica.

El desliz de un modo metonímico a un modo sinecdóquico de entender las experiencias señala un regreso a lo intrínseco. Ahora predomina una preocupación por establecer una relación entre elementos dispersos para que la entidad resultante quede, en términos cualitativos, más que una suma de sus partes. En términos cualitativos, cada parte es una réplica microcósmica del todo.

A diferencia de una noética metafórica, metonímica y sinecdóquica, una noética irónica representa una etapa que reflexiona sobre la naturaleza del lenguaje mismo. Aquí se busca señalar lo absurdo de las creencias que parodia, y se busca negar tácitamente lo que se afirma explícitamente o a la inversa.

Lo más importante para White es que por medio de estos tropos el lenguaje nos da diferentes modelos que pueden sugerir la dirección probable que el pensamiento de lectores tomará cuando tratan de dar significado a una región de experiencia aun no explicada por su tradición o su sentido común $(1978,73)$. Así, cada lector y cada escritor participa

en los procesos específicos que llevan a la creación de sentidos [y éstos] le identifican como miembro de una herencia cultural $u$ otra. En el proceso de estudiar un complejo de eventos dado, empieza a percibir una forma de relato posible que estos eventos podrian tomar o figurar. Su explicación posterior de cómo esta serie de eventos tomó la forma en que los percibe, narra su explicación en términos de cierto tipo específico de relato. (86)

La autonomía de cualquier texto asegura que habrá siempre múltiples combinaciones de lecturas posibles y que un texto logrará diferentes grados de éxito según la configuración tropológica en que se recibe.

Los cuatro tipos de noética que propone White ayudan a mediar entre perspectivas supuestamente contrarias. Sin embargo, un enfoque fenomenológico se compromete a acercarse a fenómenos tanto noemáticos como noéticos. Yo propongo que tal punto de partida en el mundo histórico lo podemos encontrar en los medios de comunicación que el discurso utiliza. Los 
medios de comunicación están entretejidos con nuestros sentidos y un cambio en un medio de comunicación señalará un cambio de configuración noética.

El medio de comunicación en que se lleva a cabo el proceso narrativo tendrá un impacto en la refiguración de un texto análogo al efecto que tienen los diferentes sentidos empleados por el proceso de la percepción en la experiencia del mundo. Siguiendo la filosofía de Marshal McLuhan, Walter J. Ong ha relacionado los modos en que la conciencia se despliega con los medios por los cuales una cultura se transmite a través del tiempo y del espacio. Hay cuatro etapas de desarrollo en los medios de comunicación cultural que presentan características semejantes a las noéticas que acabamos de examinar. Como la perspectiva metafórica, la primera etapa es la de una cultura audio-oral en la que se mantienen lazos estrechos con el ahora omnipresente. La oralidad primaria exige participación, integración y una continuidad entre un narrador como voz de la tradición y su audiencia. Con la introducción de la escritura quirográfica, una cultura se vuelve visual. Se libera de los lazos que atan la palabra hablada al aquí y ahora y se extiende hacia el espacio para explorar con mayor libertad. Esta noética visual goza de una capacidad de abstracción mayor y de reflexión analítica más precisa pero sufre de un enajenamiento social mayor. Surge una dialéctica entre culturas: las audio-orales que dependen de técnicas nemónicas para su sobrevivencia y las visuales que gozan de la escritura. Ong explica:

La vista aísla; el oído une. Mientras la vista sitúa al observador fuera de lo que está mirando, a la distancia, el sonido envuelve al oyente. Como observa Merleau-Ponty (1961), la vista divide. La vista llega a un ser humano de una sola dirección a la vez: para contemplar una habitación o un pasaje, debo mover los ojos de una parte a otra. Sin embargo, cuando oigo, percibo el sonido que proviene simultáneamente de todas direcciones: me hallo en el centro de mi mundo auditivo, el cual me envuelve, ubicándome en una especie de núcleo de sensación y existencia. Este efecto de concentración que tiene el oído es lo que la reproducción sonora de alta fidelidad explota con gran complejidad. Es posible de sumergirse en el oído, en el sonido. No hay manera de sumergirse de igual modo en la vista. $(1987,75-6)$

Este cambio de una noética oral a una noética quirográfica es un cambio hacia un conocimiento más objetivo. El conocimiento radica en objetos, en libros, ya no en la memoria de un juglar o de un sabio. La audiencia se convierte en un espectador esencialmente fuera de la acción y termina observando un reflejo mimético de los tiempos en que vive.

El pasar de una noética quirográfica a la de una conciencia tipográfica, mecaniza el lenguaje. Un libro puede aparecer en los estantes de librerías en lugares tan distantes como México, Buenos Aires, París y Nueva York el mismo día. Crece la importancia de la función hermenéutica y surge una comunidad 
académica y científica dedicada a la abstracción y al análisis de cosas, de redes conceptuales y de estructuras espaciales más que de relaciones personales. La nueva distancia facilita una mayor reflexión analítica pero crece la necesidad de integrar el nuevo conocimiento y las diferentes perspectivas en una entidad consistente.

Hoy en día los medios de comunicación electrónica nos permiten grabar nuestra voz y contemplar la paradoja que es el lenguaje. La cultura se bifurca en una oralidad secundaria con el teléfono, la televisión y el radio, por un lado, y se concentra o intensifica la tipografia en la computadora por el otro. Irónicamente, podemos llevar a cabo análisis abstractos usando lenguàs de computación cuyos programas ni entendemos y cuando oímos nuestra voz en una grabadora a nosotros no nos suena tan auténtica como les suena a otros.

Estos modos de entender y de transmitir una cultura no son momentos discretos. Se trata de una diferencia de grado y de énfasis con regiones de transición en un proceso de constante transformación. Diferentes medios pueden concurrir en el tiempo y en el espacio y resultan en una gran riqueza de perspectivas. Pero esta riqueza de perspectivas trae consigo una nueva responsabilidad hermenéutica. Si vamos a hablar de la perspectiva en la narrativa, y no solamente de la perspectiva en la narrativa que se lleva a cabo en determinadas clases sociales de ciertos países europeos del siglo veinte, tenemos que tomar en cuenta los diferentes grados de tensión que resultan de la diferencia y la semejanza entre las diferentes perspectivas que se entrelazan en la realización de un texto. Hay que tomar en cuenta los diferentes grados de tensión que resultan entre el discurso metafórico de una noética oral, como el que predomina en partes de México, y el discurso irónico de una noética tipográfica o electrónica como el que predomina en otras culturas supuestamente desarrolladas. Con este fin en mente habrá que considerar la perspectiva en la narrativa en términos de una mediación entre el lector (entendido como un conjunto de lenguaje, percepción y experiencia), el texto (entendido como un teclado de facetas, unas de la voz narrativa, otras del mundo narrativo), y el mundo de medios tecnológicos y figurativos que informan la creación y re-creación del texto. Al entrar sus diferentes facetas en juego, la perspectiva en la narrativa pasa por sus tres dimensiones de lector, texto y mundo. Las cuatro facetas de la voz narrativa (persona, dramatización, alcance, confiabilidad) más la secuencia y duración temporal median entre el fenómeno simbólico del texto y la dimensión subjetiva del lector. Esta relación se caracteriza por un diálogo de pregunta y respuesta. Mediando el texto y el campo objetivo del mundo histórico, encontramos las facetas del mundo narrativo (tipos de personajes, papeles que juegan, privilegio, y confianza) así como el campo espacial con objetos en primer plano y un trasfondo. Aquí crece una tensión en la función relacional de la cópula. El mundo narrativo no es el mundo que nos rodea pero 
sí es nuestro mundo mientras leemos. Mediando la obra en el mundo y el lector frente a su experiencia hermenéutica del texto, encontramos diferentes modos de entendimiento tropológico, ya sea metafórico, metonímico, sinecdóquico e irónico, que están unidos a diferentes medios de comunicación, ya sea de una oralidad primaria, una escritura quirográfica, una escritura tipográfica o una oralidad secundaria en conjunto con computadoras. Estos diferentes modos darán forma a la perspectiva con la que el lector se acerca al texto y resultarán en una tensión en lo que se afirma del mundo histórico. Los diferentes modos tropológicos del lenguaje y los medios por los cuales se hace sentido del mundo no pueden divorciarse de la conciencia que se extiende hacia el mundo por medio de la percepción. La percepción que tiene el lector de su mundo histórico no queda cancelada sino suspendida mientras su energía perceptiva se redirige hacia el mundo del texto. Un lector con una perspectiva metafórica tocará el teclado de facetas narrativas de una manera distinta a la de un lector con una perspectiva irónica. Las dos maneras de leer un texto son válidas pero si van a intercambiar experiencias con otros críticos de la literatura, si van a llevar a cabo lo que llamamos un estudio comparativo, tienen que estar conscientes de su punto de partida y saber que es la perspectiva en la narrativa la que las puede mediar en la configuración del texto.

Cada una de las tres dimensiones de la perspectiva en la narrativa entra en juego al realizar un texto. Así, la mediación y la tensión entre la experiencia hermenéutica que tiene el lector, la dimensión simbólica del texto y la obra como un producto de un mundo, ofrecen diferentes puntos de partida, pero puntos interrelacionados para examinar la perspectiva. Un estudio partirá de la relación entre el lector y el texto, y tratará de aclarar las voces de mundos, unos diferentes y otros semejantes. Otro explicará las estructuras que ofrecen al lector diferentes puntos de vista en el mundo del texto. Otra tradición crítica partirá de la relación entre el lector y su mundo para poder comprender las semejanzas y diferencias en una variedad de configuraciones simbólicas del texto. El punto de partida y la dirección que sigue varía según la tradición del crítico pero esa tradición crítica está también envuelta en un modo tropológico de entender el mundo y un medio de comunicación que utiliza para explicarlo. Sin embargo, central a todas las facetas de la perspectiva en la narrativa es la acción dinámica de asimilación y su movimiento correspondiente de distanciamiento, que llamamos «apropiación.»"

Los lectores perceptivos, entonces, figuran los textos por medio de diferentes grados de perspectivas metafóricas, metonímicas, sinecdóquicas e irónicas. Entran en diálogo con las facetas de la voz narrativa que se refiere a un mundo narrativo. Sin embargo, al grado que la voz dice algo significativo de algo al lector, se refiere también, aunque sea indirectamente, al mundo cotidiano en que vivimos. No sólo afecta la perspectiva que el lector tiene al leer otros textos 
sino también tiene un impacto aunque sea sutil en la perspectiva del mundo en que vive.

Entiendo, entonces, la perspectiva en la narrativa como una tipología del discurso en cuatro niveles que afectará la manera en que un autor prefigura un texto y que afectará la manera en que un lector lo refigura. Entre una perspectiva metafórica, una metonímica, una sinecdóquica y una perspectiva irónica hay lugar para múltiples combinaciones y un grado infinito de variaciones. La perspectiva en la narrativa no es únicamente la manera en que un texto se dirige a un lector. Tampoco es la proyección de una perspectiva del lector al texto. La perspectiva en la narrativa es parte de un proceso a través del cual cada lector recibe una perspectiva más amplia del mundo porque con cada lectura tiene que abrir su perspectiva a otros mundos que constituyen el objeto verdadero de toda interpretación. Por otro lado, es únicamente por medio de un texto que el lector se hace consciente de su propia perspectiva y llega a un entendimiento de sí mismo. Este entendimiento de uno mismo no es una reafirmación del ego sino una liberación de las opiniones cerradas en que la perspectiva del lector puede quedar atrapada y estancarse. La perspectiva en la narrativa es un medio hacia las experiencias literarias que revelan lo profundo del ser humano. Es un acto de recreación llevado a cabo por medio de preguntas y respuestas cuidadosas. Es también un movimiento porque mueve al lector hacia el texto y hacia un punto de partida desde donde puede reflexionar sobre su propia existencia y desde donde se le abre un mundo nuevo.

La postura teórica presentada aquí no busca estar exenta de sus propias exigencias. El esfuerzo principal de esta investigación ha sido de integrar las diferentes dimensiones de la perspectiva en la narrativa en una nueva entidad consistente y más balanceada por medio del lenguaje, la percepción, la experiencia y el concepto. Con esto en mente, se puede decir que su punto de partida está dentro de lo que Hayden White llama un modo sinecdóquico de discurso y de un modo de argumentación que busca un nuevo orden. En el hecho de reconocer la naturaleza y los límites de este punto de partida, queda descartada cualquier pretensión de clausurar la investigación a la perspectiva en la narrativa y se presenta la esperanza de haber provocado nuevas preguntas productivas para la tradición de literatura comparada que compartimos.

\section{Notas}

${ }^{1}$ Las traducciones del inglés son mías.

${ }^{2}$ Para una elaboración del concepto de mimesis, ver Paul Ricoeur (1984-1988, 1: 54-77).

${ }^{3}$ Hayden White $(1978,12)$. «Pero lo que las teorías de Piaget sí sugieren es que los tropos de figuración, metáfora, metonimia, sinécdoque e ironía, que se usan en los 
procesos conscientes de poiesis y la formación de discurso están, de algúna manera, arraigados en la herencia psicogenética del niño ... »

4 Para un desarrollo más amplio de la noción de apropiación, ver las páginas 182-193 de Paul Ricoeur (1981).

\section{Obras citadas}

Abrams, M. H. 1957. A Glossary of Literary Terms. New York: Holt, Rinehart and Winston.

Bal, Mieke. 1985. Narratology: Introduction to the Theory of Narrative. Trans. Christine van Boheemen. Toronto: $U$ of Toronto $P$.

Benveniste, Emile. 1966. Problèmes de linguistique générale. 2 vols. Paris: Gallimard.

Booth, Wayne C. 1961. The Rhetoric of Fiction. Chicago: U of Chicago P.

Borges, Jorge Luis. 1980. «Las ruinas circulares.» En Prosa Completa. 2 vols. Barcelona: Bruguera.

Forster, E. M. 1927. Aspects of the Novel. New York: Harcourt, Brace and World.

Friedman, Norman. 1967. «Distance and Point of View: An Essay in Classification.» In The Theory of the Novel. Ed. Philip Stevick. London: Collier-Macmillan.

Frye, Northrop. 1957. Anatomy of Criticism: Four Essays. Princeton: Princeton University Press.

Gadamer, Hans-Georg. 1975. Truth and Method. Trans. Garrett Barden and John Cumming. New York: Continuum.

Genette, Gerard. 1980. Narrative Discourse: An Essay in Method. Trans. James E. Lewin. Ithaca: Cornell University Press.

Humboldt, Wilhelm von. 1988. On Language: The Diversity of Human LanguageStructure and its Influence on the Mental Development of Mankind. Trans. Peter Heath. Cambridge: Cambridge University Press.

Iser, Wolfgang. 1978a. The Act of Reading: A Theory of Aesthetic Response. Baltimore: The Johns Hopkins University Press.

- 1978b. «Narrative Strategies as a Means of Communication.» In Interpretation of Narrative. Eds. Mario J.Valdés and Owen J. Miller. Toronto: U of Toronto P.

James, Henry. 1934. The Art of the Novel. New York: Charles Scribner's Sons.

Jauss, Hans Robert. 1985. «The Identity of the Literary Text in the Changing Horizon of Understanding. . In Identity of the Literary Text. Eds. Mario J. Valdés and Owen Miller. Toronto: U of Toronto P.

León-Portilla, Miguel y Angel Ma. Garibay. 1972. Visión de los vencidos: relaciones indigenas de la conquista. México: UNAM.

Lotman, Jurij. 1977. The Structure of the Artistic Text. Ann Arbor: U of Michigan P. Lubbock, Percy. 1957. The Craft of Fiction. New York: Viking Press.

Merleau-Ponty, Maurice. 1962. Phenomenology of Perception. Trans. Colin Smith. London: Routledge and Kegan Paul.

- 1964. L'Oeil et l'Esprit. Paris: Gallimard.

Ong, Walter J. 1987. Oralidady escritura: Tecnologias de las palabras. México: FCE. Ricoeur, Paul. 1977. The Rule of Metaphor: Multi-disciplinary Studies of the Creation of Meaning in Language. Trans. Robert Czerny, Kathleen McLaughlin and John Costello SJ. Toronto: U of Toronto P. 
-1981. Hermeneutics and the Human Sciences: Essays on Language, Action and Interpretation. Trans. and Ed. John B. Thompson. Cambridge: Cambridge University Press.

- 1984, 1988. Time and Narrative. 3 vols. Trans. Kathleen McLaughlin and David Pellauer. Chicago: U of Chicago P.

Rimmon-Kenan, Shlomith. 1983. Narrative Fiction: Contemporary Poetics. London: Methuen.

Scholes, Robert E. and Robert Kellogg. 1966. The Nature of Narrative. New York: Oxford University Press.

Sieber, Harry. 1978. Language and Society in La vida de Lazarillo de Tormes. Baltimore: The Johns Hopkins University Press.

Weimann, Robert. 1975. "Reception Æsthetics' and the Crisis in Literary History." CLIO. 5. 1. 3-29.

White, Hayden. 1978. Tropics of Discourse: Essays in Cultural Criticism. Baltimore: The Johns Hopkins University Press. 